\title{
Efecto del incremento de la actividad física sobre la condición física en un grupo de adolescentes con sobrepeso y/u obesidad
}

\author{
Effect of increased physical activity on physical fitness in \\ an overweight and/or obese group of adolescents
}

\author{
Isabel María Ruiz López ${ }^{1 *}$, Miguel Martín-Matillas, Manuel Delgado-Fernández ${ }^{1}$, Elena Delgado-Rico², \\ Cristina Campoy Folgoso ${ }^{3}$ y Antonio Verdejo-García ${ }^{4}$ \\ 1 Departamento de Educación Física y Deportiva, Facultad de Ciencias del Deporte, Universidad de Granada (España). \\ 2 Departamento de Psicología Clinica y Experimental, Universidad de Huelva (España). \\ 3 Departamento de Pediatría, Facultad de Medicina, Universidad de Granada (España). \\ 4 School of Psychological Sciences and Monash Institute of Cognitive and Clinical Neurosciences, Monash University (Australia).
}

Resumen: Se analiza el efecto del incremento de actividad física (AF) para conseguir el cumplimiento de recomendaciones de AF para la mejora de la salud según la Organización Mundial de la Salud en un grupo de 42 adolescentes (28 chicas) con sobrepeso/obesidad $\left(\mathrm{IMC}=29.37 \pm 4.5 \mathrm{Kg} / \mathrm{m}^{2}\right)$. El tratamiento de 12 semanas de duración consistió en la prescripción y seguimiento de AF en dos fases: intensiva (seguimiento semanal) y extensiva (seguimiento quincenal). El nivel de AF se valoró mediante el cuestionario Physician-based Assessment and Counseling y la condición física mediante pruebas de fuerza isométrica del tren superior, fuerza explosiva de piernas y capacidad cardiorrespiratoria de la batería ALPHA. Tras el tratamiento se observa una mejora significativa $(\mathrm{p}<0,05)$ en capacidad cardiorrespiratoria. En conclusión, se muestra que incrementando la práctica de AF y cumpliendo las recomendaciones semanales de 300 minutos de AF moderadavigorosa los adolescentes mejoraron su capacidad cardiorrespiratoria.

Palabras claves: Actividad Física, Condición Física, Adolescentes, Sobrepeso, Obesidad
Abstract: This study evaluated the effect of increased physical activity (PA) to achieve PA recommendations according to the World Health Organization for improving health in a group of 42 adolescents (28 girls) with overweight / obesity $\left(\mathrm{BMI}=29.37 \pm 4.5 \mathrm{Kg} / \mathrm{m}^{2}\right)$. The 12 weeks treatment consisted in the prescription and follow-up of the PA guidelines divided in two phases: intensive (weekly follow-up) and extensive (fortnightly follow-up). PA level was assessed using the Physician-based Assessment and Counseling questionnaire and the Physical Fitness using upper body isometric strength test, lower body explosive strength test and cardiorespiratory fitness test, all from the ALPHA battery fitness tests. After treatment, a significant improvement was observed $(\mathrm{p}<0.05)$ in cardiorespiratory fitness. In conclusion, this study shows that increasing PA practice and meeting the weekly recommendations of 300 minutes of moderate-vigorous PA, the adolescents' group improved their cardiorespiratory fitness.

Keywords: Physical Activity, Physical Fitness, Adolescents, Overweight, Obesity

\section{Introducción}

La inactividad física se ha convertido en el cuarto factor de contingencia de mortalidad mundial representando un elemento de riesgo del 6\% de las muertes registradas en el mundo (OMS, 2010) y aumenta el peligro de sufrir enfermedades no transmisibles como enfermedad coronaria, diabetes tipo 2, ciertos tipos de cáncer (Lee et al., 2012). Además, la inactividad física, directamente relacionada con el balance energético, es una de las causas de obesidad y de enfermedades crónicas, tanto en la población en general como específicamente en la adolescencia (Booth, Roberts \& Laye, 2012). Así, la OMS (2014), indica que el $81 \%$ de los adolescentes de edades comprendidas entre los 11 y 17 años no realiza suficiente AF, siendo este dato más alarmante en las niñas, como se ha apreciado en múltiples estudios nacionales e internacionales (Martínez, Welk, et al., 2009; OECD, 2012; Ortega, Ruiz,

Dirección para correspondencia [Correspondence address]: Isabel María Ruiz López. Universidad de Granada (España).

E-mail: iruilop@correo.ugr.es
Hurtig \& Sjöström, 2008; Ramos, Jiménez, Rivera \& Moreno, 2016).

Por otro lado, la obesidad mundial se multiplicó casi por tres entre 1975 y 2016. El incremento del sobrepeso y la obesidad entre los 5 y los 19 años ha pasado de ser de un $4 \%$ en 1975 a un $18 \%$ en 2016, no siendo muy marcada la diferencia por género. La estimación mundial de niños y adolescentes con obesidad es de 124 millones y que sufren sobrepeso u obesidad de más de 340 millones (OMS, 2016), donde uno de cada seis chicos y una de cada diez adolescentes europeas de 15 años presentaban exceso de peso en 2009-2010. Los países del sur de Europa, como España, tienen tasas de sobrepeso u obesidad a estas edades por encima del 15\% (OECD, 2012).

En una etapa en la que se producen cambios importantes a nivel de AF, comportamiento sedentario, alimentación y salud psicológica (Alberga, Sigal, Goldfield, Prud'homme \& Kenny, 2012), la calidad de vida relacionada con la salud de los jóvenes podría optimizarse, entre otros, mediante la me- 
jora de la Condición Física (CF) (Morales et al., 2013), ya que una mejor CF en la nińez y en la adolescencia se ha relacionado con una menor probabilidad de padecer sobrepeso u obesidad en la adolescencia (Ortega et al., 2011). Además, existen evidencias de que altos niveles de CF en estas etapas están asociadas a perfiles cardiovasculares saludables y a un menor riesgo de padecer enfermedades de este tipo en la vida adulta (Ruiz, 2009).

La promoción del incremento de AF desde edades tempranas, principalmente de AF moderada a vigorosa, producen mejoras tanto en la composición corporal como en la CF (Leppänen et al., 2016); por ejemplo, los adolescentes considerados activos tienen mejor capacidad cardiorrespiratoria que los inactivos según Martínez y Sánchez (2008) y una mejora de la $\mathrm{CF}$ en relación únicamente a la capacidad cardiorrespiratoria produce mejoras en el IMC y en el porcentaje de grasa corporal (Castillo \& Balaguer, 2002; Mayorga-Vega, Merino-Marban \& Rodríguez-Fernández, 2013). La capacidad cardiorrespiratoria se relaciona especialmente de manera inversa con los niveles de grasa corporal de hoy y del futuro (Ortega, Ruiz, \& Castillo, 2013). La idea de realizar AF para mejorar la CF en la infancia puede ser una estrategia eficaz para luchar contra el sobrepeso y la obesidad en la adolescencia (Ortega et al., 2011). Al respecto, se han llevado a cabo intervenciones para aumentar la AF y disminuir comportamientos sedentarios que han provocado mejoras en el IMC, evitando la obesidad (Brown \& Summerbell, 2009; López, López \& Díaz, 2016). Rocha Silva y Martín-Matillas, Carbonell-Baeza, Aparicio y Delgado-Fernández (2014) observaron en su estudio de revisión que los hallazgos de varios estudios multicomponente, en los que la combinación de AF con la orientación alimentaria generaba efectos positivos tanto en la reducción de peso como en el IMC en población infantil y adolescente. Gao et al., (2013) en su estudio mostraron como la intervención relativa a dieta y AF generó una disminución de peso en los sujetos. Su programa de AF incluía al menos una hora de actividad aeróbica estructurada pero no dirigida. A su vez, Debar et al., (2011) observaron una disminución en el IMC en un grupo de chicas adolescentes con obesidad. Su intervención, también multicomponente, marcaba objetivos de AF relativos al tiempo dedicado a la misma a lo largo de los 5 meses que duró el programa, en los que también incluyeron yoga así como elementos de ejercicios para alentar a su realización. Aunque faltan más estudios que confirmen si la AF no supervisada dentro de un programa multicomponente también produce efectos en las diferentes variables estudiadas.

En un estudio previo de nuestro grupo, se observaron mejoras en el IMC en un grupo de adolescentes que recibió una intervención multidisciplinar que combinó modificaciones en alimentación, AF y apoyo psicológico, y cuyo objetivo fue estudiar la influencia de variables neuropsicológicas implicadas en la regulación motivacional de la alimentación en el tratamiento de la obesidad juvenil (Delgado et al., 2012).

Nuestro objetivo es conocer los efectos que provoca un incremento de la AF sobre un grupo de adolescentes con sobrepeso/obesidad sobre la condición física y la composición corporal, como parte de un tratamiento multicomponente (AF, Alimentación y apoyo psicológico) de 12 semanas de duración.

\section{Método}

\subsection{Muestra}

En este estudio han participado un total de 42 adolescentes (14 hombres y 28 mujeres). El rango de edad fue de 12-17 años. La media \pm desviación estándar (DE) fue de 14,21 $\pm 1,3$ años. El valor medio inicial del Índice de Masa Corporal $\left(\mathrm{IMC}=\operatorname{peso}(\mathrm{kg}) / \mathrm{talla}^{2}(\mathrm{~m})\right)$ fue de $29.15 \pm 4.5 \mathrm{Kg} / \mathrm{m}^{2}$. La elección de la muestra se hizo en centros educativos y en la Unidad de Endocrinología del Hospital "Virgen de las Nieves" de Granada (Espańa), con el fin de participar en un estudio multidiciplinar para adolescentes con exceso de peso (Estudio BRAINOBE).

Los adolescentes participantes tenían que cumplir con los siguientes criterios: (a) edad entre 12 y 17 años; (b) IMC entre los niveles de sobrepeso y obesidad de acuerdo con el International Obesity Task Force (IOTF) que definen Cole et al., (2000); (c) ausencia de evidencia en el pasado o en el presente de desórdenes psicológicos; (d) firma del consentimiento informado por parte de los padres y la implicación activa durante el proceso de al menos uno de los padres. Los padres tuvieron que firmar un documento dando su consentimiento para que los adolescentes participaran en el programa. El presente estudio forma parte del proyecto BRAINOBE, destinado a probar una intervención conductual multidisciplinar para jóvenes con exceso de peso, cuyo principal objetivo es modificar los estilos de vida relacionados con la alimentación y mejorar tanto las habilidades cognitivo-conductuales como la actividad física. El estudio fue aprobado por el Comité Ético para la Investigación Humana de la Universidad de Granada. Todos los procedimientos se llevaron a cabo de conformidad con la Declaración de Helsinki.

En la figura 1 se muestra el diagrama de flujo de los participantes del estudio. De los 43 participantes contactados inicialmente, sólo 42 comenzaron la intervención ya que uno excedía el percentil de peso que se había marcado como requisito. La $\mathrm{N}$ final se redujo a 39 por abandono. 


\section{Excluido n=1 (Obesidad Mórbida)}

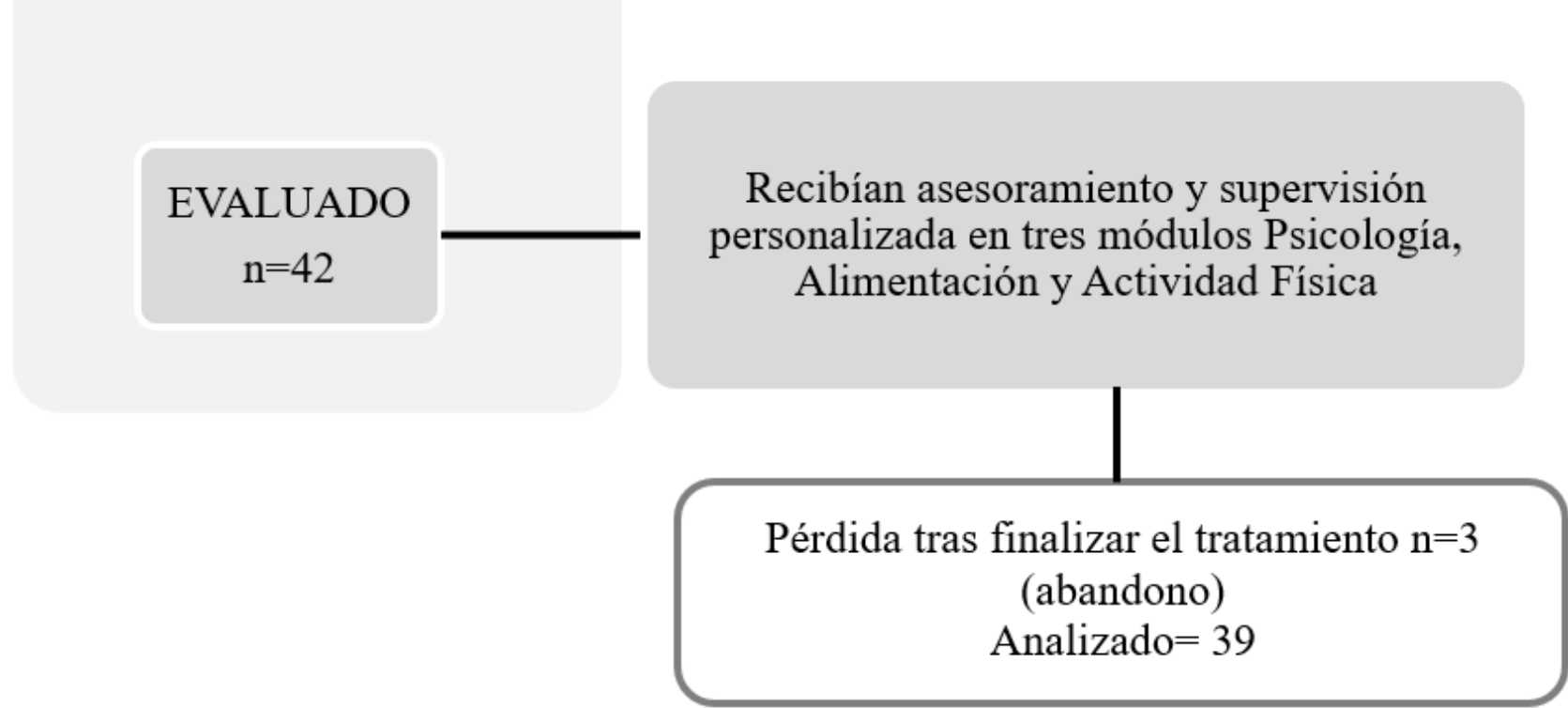

Figura 1. Diagrama de flujo del estudio.

\subsection{Diseño}

Se empleó un diseño longitudinal de intervención con dos medidas de evaluación: antes y después del tratamiento. Las medidas iniciales evaluadas fueron IMC, mediciones de condición física y de actividad física. La intervención multicomponente y multidisciplinar tuvo una duración de 12 semanas.

\subsection{Material}

\section{Análisis biomédicos}

Índice de masa corporal. El IMC se obtuvo a partir de medidas directas de peso y talla y se calculó para cada participante como la relación peso $(\mathrm{Kg}) /$ talla $\left(\mathrm{m}^{2}\right)$.

Porcentaje de grasa corporal. Medición realizada a través de bioimpedancia o impedancia bioeléctrica. Responde a la ecuación Masa grasa= Peso- Masa muscular (\%).

Evaluación de la condición física

Para la evaluación de la CF fueron seleccionadas tres pruebas integradas dentro de la batería ALPHA (Ruiz et al., 2011). Las pruebas de CF realizadas tienen asociadas los valores de referencia de la CF de los adolescentes españoles, lo que permite evaluar el nivel de los participantes (Ortega et al., 2005). Las tres pruebas seleccionadas para este estudio fueron:

Test de Course Navette o test de ida y vuelta de $20 \mathrm{~m}$ (Lèger, Mercier, Gadoury \& Lambert, 1988) que mide la capacidad aeróbica, siendo una medida directa del estado fi- siológico de la persona (Ruiz et al., 2006). A partir de esta medida e incluyendo la edad (E) y la velocidad final $(\mathrm{V}=8+$ $0.5 \mathrm{x}$ último estadio completado) se obtuvo el VO2máx, que se define como el máximo consumo que se alcanza durante un ejercicio máximo hasta el agotamiento voluntario.

$\mathrm{Vo}^{2}$ Máx $=(31,025)+(3,238 \mathrm{~V})-(3,248 \mathrm{E})+(0,1536 \mathrm{VE})$

La distancia de 20 metros en sus extremos deberá quedar claramente seńalada. La prueba se realiza una sola vez. Se registra el último medio palier o estadio completado por el participante. La distancia entre los mismos debe garantizar al menos un metro. El test consiste en ir y volver corriendo una distancia de 20 metros a la velocidad que va emitiendo una señal de audio a intervalos regulares de un minuto. Esa velocidad se incrementa $0,5 \mathrm{Km} / \mathrm{h} / \mathrm{min}$ de manera constante comenzando a velocidad de $8,5 \mathrm{Km} / \mathrm{h}$. Una vez escuchada la primera señal, el participante se dirigirá al extremo opuesto antes de que vuelva a escuchar la señal. El objetivo es mantener el ritmo durante el mayor tiempo posible completando el mayor número de palieres seguidos.

Dinamometría de prensión manual. Se evalúa la fuerza máxima de flexores palmares de la mano a través de un dinamómetro digital con agarre ajustable (Modelo TKK 5101 Grip D; Takey, Tokyo, Japan) y una regla-tabla para medir la mano y calcular la distancia de agarre del dinamómetro. Previamente se debe medir el tamaño de una de las manos extendida, midiendo la distancia que separa los extremos distales del dedo pulgar y meńique. El participante aprieta lentamente y de manera continua el dinamómetro durante al 
menos 2 segundos. El codo estará extendido y no se permite el contacto del dinamómetro con otra parte del cuerpo que la mano con la que se ha iniciado la evaluación. El test se realiza dos veces con cada mano y alternativamente con ambas manos. Se tendrá en cuenta el mejor valor para cada mano cuya medida se valora en kilogramos $(\mathrm{kg})$.

Salto de longitud a pies juntos. Evalúa la fuerza explosiva de miembros inferiores a través de un salto con impulso simultáneo de ambos pies desde la línea de salto. Se empleó una cinta métrica, cinta adhesiva y conos. Desde posición de pie, situado detrás de la línea y con ambos pies a la misma altura y a la anchura de los hombros, con ayuda del balanceo de los brazos, se realizará un salto hacia adelante intentando alcanzar la mayor distancia posible. Se valorará el mejor de dos intentos medidos en centímetros $(\mathrm{cm})$ siendo la distancia máxima la que marca la línea de salto y la parte posterior del pie más próximo a dicha línea.

\section{Evaluación de la actividad física}

El nivel de AF del adolescente, respecto a la recomendación específica para adolescentes de realizar al menos 60 minutos de AF 5 días a la semana, se evaluó a través del cuestionario de actividad física PACE (Martínez et al., 2009; Prochaska, Sallis \& Long, 2001). Los participantes responden a la pregunta: En una semana normal, ¿cuántos días haces AF 60 minutos o más? Marcando un valor que va desde Ninguno (0) hasta Seis o más (6+). Previamente y a modo introductorio se define qué es la AF y se aportan ejemplos de la misma. Esa consideración permite agruparlos en inactivos (menos de tres días), insuficientemente activos (entre tres y cinco días) y activos (más de cinco) y así poder programar su AF en cuanto a frecuencia semanal. Este cuestionario fue inicialmente validado en inglés (Prochaska et al., 2001) y posteriormente en espańol (Martínez, Martínezet al., 2009).

Intervención del tratamiento multidisciplinar

La intervención se hizo en grupos de 10-12 participantes cada uno, y se intervino durante doce semanas consecutivas en una sesión semanal. Se llevó a cabo en cuatro grupos, en oleadas, hasta completar un total de 42 adolescentes. La intervención incluyó tres módulos: (a) un módulo psicosocial donde en talleres semanales se trabajó la formación de habilidades específicas en las que se incluía tanto habilidades cognitivas (control inhibitorio, planificación y resolución de conflictos) como afectivas (expresión emocional y regulación); (b) un módulo de alimentación que consistió en la prescripción y seguimiento de dietas personalizadas en función del sexo, edad y las puntuaciones z-score del IMC (Moreno et al., 2006). El seguimiento semanal se hizo con la nutricionista y la psicóloga durante las citas personales con los adolescentes y sus padres. El control alimentario se realizó mediante entrevistas y la revisión del control dietético para facilitar la observación objetiva (Martínez-Gómez et al., 2009); (c) un módulo de actividad física que consistió en la prescripción de un programa de AF personalizado cuyo objetivo era lograr el ejercicio mínimo establecido para este grupo de edad que es de $1 \mathrm{~h} / \mathrm{día}$, al menos 5 días a la semana (WHO, 2010; Martínez- Gómez et al., 2009). El ejercicio se prescribió inicialmente en $1 \mathrm{~h}$ de ejercicio aeróbico de intensidad moderada a vigorosa de 3 a 5 días/ semana, en función del nivel inicial de cada persona. El gasto energético se estimó en valores equivalentes metabólicos (Ainsworth et al., 2011; Ainsworth et al., 2000) para cada actividad y la frecuencia e intensidad de las actividades del programa de ejercicios. El intervalo de gasto energético estimado fue de 15 a $23 \mathrm{Kcal} / \mathrm{Kg}$ de peso corporal/ semana.

El programa de intervención de 12 semanas de duración, en cuanto a la AF se refiere, incluyó dos etapas: una fase intensiva donde la revisión de la AF tenía lugar semanalmente, y otra extensiva donde la estimación se realizó cada dos semanas. En la primera sesión, tras la medición inicial de las diferentes variables de la $\mathrm{CF}$ y de la $\mathrm{AF}$, se prescribió el ejercicio en base a su perfil de AF y su evaluación de la CF. La valoración de la CF a través del nivel de capacidad cardiorrespiratorio determinó si cada participante podía o no realizar AF vigorosa (solo aquellos participantes con niveles altos o muy altos según Ruiz et al., (2011). A partir de todos estos datos, se programó el ejercicio que el adolescente se comprometió a realizar a lo largo de una semana. Cualquier participante pudo incrementar más cantidad de AF siempre y cuando: (i) existiera un buen cumplimiento; (ii) no apareciese fatiga excesiva debido al incremento de AF. En esta sesión familiar, además de la prescripción, se proyectó información general sobre AF tal como definición, beneficios de su práctica y se dio una visión general del seguimiento a lo largo del tratamiento. A partir de esta sesión y en un total de 6 semanas, se citó a los mismos con el fin de valorar el grado de cumplimiento y así poder establecer las modificaciones pertinentes para la semana siguiente. Al final de esta etapa se realizó una puesta en común tanto con los adolescentes como con las familias ajustando los objetivos y facilitando estrategias para ser activos y superar barreras para poder llevar a cabo su plan de AF (Martínez-Gómez et al., 2009). El objetivo se modificó para todos aquellos que inicialmente tenían que cumplir al menos tres días 1 hora de AF hasta conseguir al menos cinco días. En las seis semanas restantes, la valoración de la AF se hizo cada dos semanas. Al final de este periodo y coincidiendo con la semana 12 se repasaron los puntos clave. La AF se fue incrementando en la medida que se cumplían los objetivos, evitando la fatiga excesiva y reduciendo las conductas sedentarias. Tras el periodo de seguimiento de 12 semanas de duración, se realizó la evaluación. 


\begin{tabular}{|c|c|c|c|}
\hline Tratamiento & & \multicolumn{2}{|l|}{ Evaluación } \\
\hline Pre-Test & Sesión 0 & \multicolumn{2}{|l|}{$\begin{array}{l}\text { Condición Física (Test de batería ALPHA) } \\
\text { Cuestionario de AF } \\
\text { IMC }\end{array}$} \\
\hline \multirow[t]{6}{*}{$\begin{array}{l}\text { Fase Intensiva } \\
\text { (6 semanas) }\end{array}$} & Sesión 1 & $\begin{array}{l}\text { Prescripción de AF según nivel de AF y valoración de la CF para deter- } \\
\text { minar la idoneidad de incluir o no AF vigorosa }\end{array}$ & Taller familiar sobre AF \\
\hline & Sesión 2 & \multirow{4}{*}{\multicolumn{2}{|c|}{ Seguimiento semanal }} \\
\hline & Sesión 3 & & \\
\hline & Sesión 4 & & \\
\hline & Sesión 5 & & \\
\hline & Sesión 6 & Modificación del plan & Taller familiar sobre AF \\
\hline \multirow{3}{*}{$\begin{array}{l}\text { Fase Extensiva } \\
\text { (6 semanas) }\end{array}$} & Sesión 8 & \multirow{2}{*}{\multicolumn{2}{|c|}{ Seguimiento quincenal }} \\
\hline & Sesión 10 & & \\
\hline & Sesión 12 & \multicolumn{2}{|l|}{ Taller resumen familiar de los aspectos claves para seguir siendo activo } \\
\hline Post-Test & & \multicolumn{2}{|l|}{$\begin{array}{l}\text { Condición Física (Test de batería ALPHA) } \\
\text { Cuestionario de AF } \\
\text { IMC }\end{array}$} \\
\hline
\end{tabular}

Figura 2. Diseño del programa de AF.

\subsection{Análisis estadístico}

Se ha realizado un análisis estadístico por medio del programa SPSS versión 21 (IBM SPSS Statistics for Windows, version 22.0, Armonk, NY). Los resultados se presentan como media desviación típica. Se aplicó la prueba de bondad de Kolmogorov-Smirnov para comprobar que los resultados seguían una distribución normal y en su defecto Shapiro-Wilk. Para variables con comportamiento no paramétrico (no cumplían la distribución normal) se empleó la prueba de Wilcoxon con el fin de determinar si existía diferencia en el post-test respecto al pre-test, para el último periodo recorrido en la prueba de Course Navette y el salto horizontal a pies juntos. Para variables con comportamiento paramétrico (distribución normal), VO2máx y dinamometría manual, se utilizó la prueba $T$ para muestras relacionadas. Al relacionarse directamente el consumo máximo de oxígeno con el último periodo completado de la prueba de Course Navette se consideró la necesidad de comprobar su significatividad a través de la prueba alternativa a la de Kolmogorov-Smirnov. A su vez, se halló la $d$ de Cohen como medida del tamaño del efecto, y se interpretó como pequeño $(\sim 0.2)$, medio $(\sim 0.5)$ o grande ( 0.8 o más) (Nakagawa, S. \& Cuthill. I. C., 2007). También se analizaron los datos diferenciando por sexos y se empleó la t de student (prueba paramétrica) y la U de Mann- Whitney (no paramétrica) para muestras independientes para compararlos entre sí. Los resultados eran considerados estadísticamente significativos para $\mathrm{p}<0.05$.

\section{Resultados}

Se evaluaron 42 adolescentes, 28 chicas $(66.7 \%)$ y 14 chicos (33\%). La tabla 1 muestra las variables sociodemográficas, talla, peso, IMC, porcentaje de grasa corporal y de condición física general del grupo y diferenciado por género al inicio del programa. Los datos presentan el intervalo de valores junto a su media y desviación típica. El grupo se encuentra en un rango de edad entre los 12- 17 años, y muestra valores más altos respecto al peso, la altura y el IMC en chicos, no ocurre así en relación al porcentaje de grasa corporal. Todas las medidas, excepto la altura, que describe un valor significativo, se inclinan hacia la significación. Los valores que evalúan la CF son más destacados en chicos que en chicas. Existe significancia tanto en la fuerza explosiva del tren inferior como en el VO2 máx, hallado a través de la prueba de Course Navette. La fuerza de prensión manual y la prueba de Course Navette tienden a la significación.

En la Tabla 2 se describe la comparación de medias de IMC y variables de CF entre el pre-test y post-test y su grado de significación para el grupo total. Se aprecian disminuciones significativas en peso (>4 kg), IMC, $\%$ de grasa corporal y fuerza de prensión manual, e incrementos significativos en talla $(>1 \mathrm{~cm})$, capacidad aeróbica y VO2max. En el IMC y $\%$ de grasa corporal se aprecia un tamaño del efecto medio calculado con d de Cohen. Respecto a las variables evaluadas de CF, se produjo una pérdida de fuerza y un cambio positivo significativo en la prueba de $\mathrm{CN}$, en relación a los paliers conseguidos, y al $\mathrm{VO}^{2}$ max hallado respecto a dicha prueba $(\mathrm{p}<0.05)$.

En la Tabla 3 se muestra la comparación entre medidas pretest y postest de las variables de talla, peso, IMC, porcen- 
taje de grasa corporal y CF diferenciadas por género. En todos los casos se aprecia una disminución de peso, del IMC y del porcentaje de grasa corporal y un incremento de la altura. El grupo de chicas sufrió una disminución de peso próxima a cinco $\mathrm{Kg}$ en relación a los algo más de $3 \mathrm{Kg}$ y medio de pérdida que sufrieron los chicos. El IMC varió de manera similar en ambos grupos ( $d$ de Cohen medio en chicas y de 0,41 en chicos). El porcentaje de grasa corporal fue algo más destacado en chicos ( $\mathrm{d}$ de Cohen de 0,48 frente al 0,97 de las chicas),

Respecto a la CF, las chicas muestran unos niveles en todas las pruebas evaluadas inferiores respecto a los chicos, tendencia que permanece tras la intervención. El componente de la fuerza cuya valoración se hizo a través de las pruebas de dinamometría y salto horizontal, sufre un descenso similar en ambos grupos tras las doce semanas que duró el estudio. En ambos casos sufren un tamaño del efecto pequeño. En relación a la capacidad cardiorrespiratoria medida a través de la prueba de Course Navette se observa una ligera mejora en relación al pre-test o línea base en ambos grupos, más destacada en chicos. Lo mismo ocurre con el Vo2 máx, hallado a través del último palier completado en la prueba de $\mathrm{CN}$, no llegando al milímetro de oxígeno de mejora. En ningún caso ni para ningún sexo hay significatividad.

Tabla 1. Características descriptivas al inicio del estudio

\begin{tabular}{|c|c|c|c|c|c|c|c|c|c|c|}
\hline \multirow[b]{2}{*}{ Medidas } & \multicolumn{3}{|c|}{ TOTAL } & \multicolumn{3}{|c|}{ CHICAS } & \multicolumn{3}{|c|}{ CHICOS } & \multirow[t]{2}{*}{$\mathrm{P}$ valor } \\
\hline & $\mathrm{n}$ & Rango & Media (d.t.) & n & Rango & $\begin{array}{c}\text { Media } \\
\text { (d.t.) }\end{array}$ & $\mathrm{n}$ & Rango & Media (d.t.) & \\
\hline Edad (años) & 42 & $12-17$ & $14.21 \pm 1.33$ & 28 & $12-17$ & $14.32 \pm 136$ & 14 & $12-16$ & $14.00 \pm 1.30$ & 0.469 \\
\hline Peso $(\mathrm{Kg})$ & 41 & $52-108$ & $77.10 \pm 15.07$ & 28 & $57-108$ & $74.29 \pm 13.3$ & 13 & $52-106$ & $83.15 \pm 17.31$ & 0.079 \\
\hline Altura (cm) & 42 & $148-177$ & $162.07 \pm 7.27$ & 28 & $148-173$ & $160.18 \pm 6.08$ & 14 & $151-177$ & $165.86 \pm 8.18$ & $0.015^{*}$ \\
\hline $\operatorname{IMC}\left(\mathrm{Kg} / \mathrm{m}^{2}\right)$ & 41 & $23-39$ & $29.37 \pm 4.51$ & 28 & $24-38$ & $28.93 \pm 4.28$ & 13 & $23-39$ & $30.31 \pm 5.00$ & 0.369 \\
\hline $\begin{array}{l}\text { Porcentaje de grasa corpo- } \\
\text { ral (\%) }\end{array}$ & 39 & $19-52$ & $35.56 \pm 7.92$ & 28 & $28-52$ & $37.57 \pm 5.68$ & 13 & $19-50$ & $31.23 \pm 10.32$ & 0.055 \\
\hline \multicolumn{11}{|l|}{ Medidas de CF } \\
\hline Dinanometría (Kg) & 42 & $20.4-49.8$ & $30.25 \pm 6.07$ & 28 & $20.40-37.40$ & $28.72 \pm 4.26$ & 14 & $22.30-49.80$ & $33.33 \pm 7.97$ & 0.059 \\
\hline Salto Horizontal $(\mathrm{cm})$ & 41 & $94-202$ & $133.88 \pm 24.87$ & 27 & $94-160$ & $124.78 \pm 16.72$ & 14 & $110-202$ & $151.43 \pm 28.98$ & $0.005^{*}$ \\
\hline $\begin{array}{l}\text { Course Navette } \\
\text { (último palier completado) }\end{array}$ & 41 & $2-7$ & $2.76 \pm 1.26$ & 27 & $2-5$ & $2.41 \pm 0.74$ & 14 & $2-7$ & $3.43 \pm 1.74$ & 0.06 \\
\hline VO2máx $(\mathrm{ml} / \mathrm{min} / \mathrm{Kg})$ & 41 & $27-46$ & $35.77 \pm 3.97$ & 27 & $27-42$ & $34.72 \pm 3.42$ & 14 & $30.8-46.0$ & $37.80 \pm 4.35$ & $0.017^{*}$ \\
\hline
\end{tabular}

VO2máx =Consumo Máximo de Oxígeno (Lèger, Mercier, Gadoury \& Lambert, 1988).

Los valores $\mathrm{p}<0.05$ se indican con asterisco.

Tabla 2. Efectos de la intervención en las variables estudiadas en los adolescentes.

\begin{tabular}{|c|c|c|c|c|c|c|c|}
\hline \multirow[b]{2}{*}{ Medidas } & \multicolumn{7}{|l|}{ TOTAL } \\
\hline & Pre-Test & $\begin{array}{c}\text { Pre-Test } \\
\mathrm{N}\end{array}$ & Post-Test & $\begin{array}{c}\text { Post-Test } \\
\mathrm{N}\end{array}$ & Dif. media & Signif & $d$ de Cohen \\
\hline Peso $(\mathrm{kg})$ & $77.69 \pm 15.22$ & & $73.28 \pm 15.06$ & 39 & -4.41 & $0.000^{*}$ & 0.29 \\
\hline Talla (cm) & $162.13 \pm 7.45$ & 40 & $163.45 \pm 7.25$ & 39 & 1.32 & $0.000^{*}$ & -0.17 \\
\hline $\mathrm{IMC}(\mathrm{Kg} / \mathrm{m} 2)$ & $29.59 \pm 4.51$ & 39 & $27.33 \pm 4.45$ & 39 & $-2,26$ & $0.000^{*}$ & 0.5 \\
\hline Porcentaje de Grasa Corporal (\%) & $35.74 \pm 8.05$ & 39 & $31.51 \pm 7.81$ & 39 & -4.23 & $0.000^{*}$ & 0.53 \\
\hline Dinanometría (Kg) & $29.76 \pm 5.43$ & 39 & $28.20 \pm 6.64$ & 39 & -1.56 & $0.014^{*}$ & 0.25 \\
\hline Salto Horizontal $(\mathrm{cm})$ & $132.29 \pm 24.96$ & 38 & $132.11 \pm 24.92$ & 39 & -0.18 & 0.959 & 0.007 \\
\hline Course Navette (último palier completado) & $2.68 \pm 1.23$ & 38 & $3.21 \pm 1.37$ & 39 & 0.52 & $0.002^{*}$ & -0.40 \\
\hline Vo2máx (ml/min/Kg) & $35.56 \pm 3.94$ & 38 & $36.38 \pm 4.47$ & 39 & 0,81 & $0.046^{*}$ & -0.19 \\
\hline
\end{tabular}

IMC. Índice de masa corporal (kilogramos/metros al cuadrado). Porcentaje de masa corporal medido a través de bioimpedancia (Porcentaje). Dinamometría. Media de ambas manos en Kilogramos. Salto horizontal (centímetros). Course Navette (último palier completado). Volumen máximo de oxígeno (mililitros/minuto/Kilogramos) (Lèger, Mercier, Gadoury \& Lambert, 1988).

Los valores muestran la media \pm desviación estándar. Los valores significativos se muestran con asterisco $\left(^{*}\right)$. 
Tabla 3. Efectos de la intervención en las variables estudiadas por género

\begin{tabular}{|c|c|c|c|c|c|c|c|c|c|c|}
\hline & CHICAS & & & & & CHICOS & & & & \\
\hline Medidas & $\begin{array}{l}\text { Pre-Test } \\
(\mathrm{n}=26)\end{array}$ & $\begin{array}{l}\text { Post-Test } \\
(\mathrm{n}=26)\end{array}$ & $\begin{array}{c}\text { Dif. } \\
\text { media }\end{array}$ & Signif & $\begin{array}{l}d \mathrm{de} \\
\text { Cohen }\end{array}$ & $\begin{array}{l}\text { Pre-Test } \\
(\mathrm{n}=13)\end{array}$ & $\begin{array}{l}\text { Post-Test } \\
(\mathrm{n}=13)\end{array}$ & $\begin{array}{l}\text { Dif. } \\
\text { media }\end{array}$ & Signif & $\begin{array}{l}d \text { de } \\
\text { Cohen }\end{array}$ \\
\hline Peso (kg) & $74.96 \pm 13.61$ & $70.15 \pm 13.51$ & -4.81 & $0.000^{*}$ & 0.35 & $83.15 \pm 17.31$ & $79.54 \pm 16.57$ & -3.61 & $0.001^{*}$ & 0.21 \\
\hline Talla $(\mathrm{cm})$ & $160.12 \pm 6.30$ & $161.08 \pm 5.97$ & 0.96 & $0.000^{*}$ & -0.15 & $165.86 \pm 8.18$ & $167.86 \pm 7.56$ & 2.00 & $0.000^{*}$ & -0.25 \\
\hline $\mathrm{IMC}(\mathrm{Kg} / \mathrm{m} 2)$ & $29.23 \pm 4.30$ & $26.88 \pm 4.14$ & -2.34 & $0.000^{*}$ & 0.55 & $30.31 \pm 5.00$ & $28.23 \pm 5.06$ & -2.07 & $0.000^{*}$ & 0.41 \\
\hline $\begin{array}{l}\text { Porcentaje de Grasa Cor- } \\
\text { poral (\%) }\end{array}$ & $38.00 \pm 5.61$ & $34.08 \pm 1.02$ & -3.92 & $0.000^{*}$ & 0.97 & $31.23 \pm 10.32$ & $26.38 \pm 9.64$ & -4.85 & $0.000^{*}$ & 0.48 \\
\hline Dinanometría (Kg) & $28.62 \pm 4.40$ & $27.04 \pm 4.60$ & -1.57 & $0.000^{*}$ & 0.35 & $32.06 \pm 6.67$ & $30.51 \pm 9.32$ & -1.55 & 0.387 & 0.19 \\
\hline Salto Horizontal (cm) & $122.96 \pm 15.86$ & $122.32 \pm 15.78$ & -0.64 & 0.746 & 0.04 & $150.23 \pm 29.80$ & $150.92 \pm 28.86$ & -0.69 & 0.874 & -0.02 \\
\hline $\begin{array}{l}\text { Course Navette } \\
\text { (último palier completado) }\end{array}$ & $2.36 \pm 0.70$ & $2.88 \pm 1.01$ & 0.52 & 0.16 & -0.59 & $3.31 \pm 1.75$ & $3.85 \pm 1.77$ & 0.53 & $0.047^{*}$ & -0.30 \\
\hline Vo2máx $(\mathrm{ml} / \mathrm{min} / \mathrm{Kg})$ & $34.51 \pm 3.26$ & $35.26 \pm 4.54$ & 0.78 & 0.155 & -0.18 & $37.59 \pm 4.46$ & $38.46 \pm 3.65$ & 0.87 & 0.144 & -0.21 \\
\hline
\end{tabular}

IMC. Índice de masa corporal (kilogramos/metros al cuadrado). Porcentaje de masa corporal medido a través de bioimpedancia (Porcentaje). Dinamometría. Media de ambas manos en Kilogramos. Salto horizontal (centímetros). Course Navette (último palier completado). Volumen máximo de oxígeno (mililitros/minuto/Kilogramos) (Lèger, Mercier, Gadoury \& Lambert, 1988).

Los valores muestran la media \pm desviación estándar. Los valores significativas se muestran con asterisco $\left(^{*}\right)$

\section{Discusión}

El presente estudio sobre el tratamiento del sobrepeso/obesidad en adolescentes, muestra que una intervención multidisciplinar que combina AF para alcanzar 300 min de AF de intensidad moderada a vigorosa (AFMV), pautas de alimentación y apoyo psicológico de 12 semanas produjo una disminución del peso, del IMC y, del porcentaje de grasa corporal, así como una disminución de la fuerza de prensión manual principalmente en chicas y un ligero incremento de la capacidad cardiorrespiratoria medida de manera indirecta teniendo en cuenta el último palier completado en el test de Course Navette, principalmente en chicos. La fuerza del tren inferior valorada mediante el test de salto horizontal se mantuvo constante.

Realizar una intervención multidisciplinar en la que se incluya AF con otras variables para tratar el sobrepeso y obesidad se ratifica en multitud de estudios. Así, Vasques et al., (2014) realizaron un metaanálisis con la finalidad de valorar qué efectos tenían los programas de intervención en la variable IMC tanto en niños como en adolescentes. Concluyeron que para adolescentes de entre 15 y 19 años, las intervenciones que incluían educación nutricional y de 3 a 5 sesiones por semana de AF, obtuvieron los mejores resultados. Vanhelst, Fardy, Béghin, Bui-Xuan y Mikulovic (2015) valoraron los efectos de la edad y el tipo de AF sobre las medidas antropométricas en 37 niños obesos cuya edad oscilaba entre $12,5 \pm 2,5$ que participaban en un programa de intervención único de AF y de educación para la salud. Tras la intervención hallaron reducciones significativas del IMC y concluyeron la importancia de adaptar la AF al grupo de edad para favorecer la eficacia de la intervención. La edad también se constituye como una variable importante a la hora de planificar el tipo de ejercicio (Graf et al., 2014; Vanhelst, Fardy, Béghin, Bui-Xuan \& Mikulovic, 2015).

Aguilar y Cordero et al., (2014) también comprobaron la eficacia del aumento de la AF en la reducción del sobrepeso y obesidad en nińos y adolescentes. Y es que los adolescentes españoles están lejos de cumplir las recomendaciones establecidas para su grupo de edad, tal y como demuestran los valores de AF de los adolescentes españoles participantes en el estudio Health Behaviour in School-aged Children (HBSC) de las ediciones del 2002 y 2006 publicadas en 2016 (Ramos, Jiménez, Rivera \& Moreno, 2016). El incremento de AF a lo largo del estudio realizado, junto a los componentes psicológicos y de nutrición, determinaron que la totalidad de nuestro grupo y por género, redujera el IMC $(\mathrm{p}<0.05)$ con un efecto de tamaño medio ( $d$ de Cohen=0.5), aunque siguieran estando en el rango de sobrepeso/obesidad (Chicas IMC: 26.88 \pm 4.14 ; Chicos IMC: $28.23 \pm 5.06)$. Y es que, aunque Aguilar et al., (2015) también valoran los beneficios que sobre el sobrepeso y la obesidad tienen los tratamientos que incluyen AF y dieta, también determinaron ser precavidos en el efecto rebote que un tratamiento a corto plazo puede ocasionar en las pérdidas que se produzcan durante la intervención y estimando la duración óptima de intervención mayor al año. Así, la intervención de ejercicio aeróbico por un lado y, por otro, de ejercicios combinados de fuerza, disminuyeron el IMC en un programa de 16 semanas sobre adolescentes con obesidad (Lee et al., 2010), pudiendo evitar de esa manera el efecto rebote al incrementar la masa muscular al mismo tiempo que reduce la grasa (Aguilar et al., 2015). A pesar del 
registro de 40 estudios efectuado por López, Audisio y Berra (2010) cuya intervención implicaba AF, nutrición y educación, y que mostraba eficacia en el control del peso corporal, esa eficacia se presentaba como débil, ya que la mayoría de los estudios no hallaron el resultado esperado, fueron heterogéneos y de corta duración. Tal y como hemos dicho, nuestro estudio de 12 semanas de duración, mostró mejoras sobre el IMC y el peso corporal del grupo participante, conocer las modificaciones que se establecieron en el tiempo tras la intervención se convierte en una variable a considerar para futuras intervenciones. Es preciso también señalar resultados similares encontrados en otros estudios considerados de corta duración. En un programa de tan solo 2 semanas de duración en el que se incluían dieta intensiva y ejercicio sobre un grupo de niños y adolescentes con sobrepeso u obesidad, Roberts, Izadapanah, Angadi \& Barnard (2013) encontraron una reducción de peso significativa pero no lo suficiente para que dejaran de sufrir obesidad en el grupo participante, aunque determinaron que este tipo de programas era eficaz para todos los participantes para mejorar los factores de riesgo metabólico. Así, según la revisión de programas de prevención de la obesidad realizada por Summerbell et al., (2005) se mostraba que, a pesar de que la mayoría de ellos era de corta duración (12 semanas), se encontraban pequeñas mejoras significativas del IMC. En estos programas también se incluían aspectos de dieta o de AF. Ampliando el trabajo de Summerbell et al., Waters et al., (2011) detectaron la eficacia sobre la adiposidad de los programas de intervención de otros 36 estudios más. La edad máxima de los grupos era de 18 años y la duración mínima del estudio de 12 semanas. También 12 semanas duraba el estudio de 35 escolares de Murcia (12-15 años) en los que se comprobó la relación entre la realización de AF intensa tres veces por semana y la reducción de masa grasa e IMC. En esta misma dirección, en el estudio realizado por Patrick, Gregory, Norman, Calfas et al., (2004) tan sólo la AF vigorosa pudo considerarse eficaz en la variable peso tanto en los chicos como las chicas participantes en el estudio que tuvo como objetivo examinar cómo la dieta, la AF y los comportamientos sedentarios afectaban al nivel de sobrepeso en un total de 878 adolescentes. En relación a la intensidad de la AF, en nuestro estudio el seguimiento que se realizó se hizo sobre el tiempo dedicado a la AFMV y, tan solo a aquellos adolescentes que poseían un determinado perfil de CF, basado en la medición del CN, se les asesoró para que integraran AF vigorosa. Aun así, hubo una reducción de peso significativo $(\mathrm{p}<0.05)$ aunque con un efecto de tamaño pequeño $(d$ de Cohen $=0.29)$.

Nuestro estudio realizaba un asesoramiento y seguimiento de la AF principalmente de resistencia cardiorrespiratoria, componente psicológico y nutrición, donde observamos una disminución significativa del porcentaje de grasa corporal con un tamaño de efecto de valor medio ( $d$ de Cohen=0.50), más acentuado en chicas $(\mathrm{d}=0.97)$. En contraposición, Pastor, Gil, Tortosa y Martínez (2012) realizaron una intervención en 38 alumnos de $1^{\circ}$ de E.S.O. que se encontraban por encima del percentil $85 \mathrm{sin}$ asesoramiento dietético pero con intervención programada y dirigida de AF, tras la cual se produjo una mejora en los valores de grasa corporal. La línea de un estudio realizado en Rio Branco (Brasil) fue un programa dirigido de AF basado en un trabajo de la resistencia aeróbica, fuerza resistencia y flexibilidad y, de una segunda parte de deportes de equipo, en contraposición a los que recibían el currículo propio de educación física (EF), en dicho estudio, los resultados mostraron una disminución del porcentaje de grasa corporal en 195 escolares entre los 15 y los 17 años (Dos Santos, Gonçalves, Morcillo, Guerra-Júnior \& Amancio, 2015). Cabe pensar que la intervención dietética junto el apoyo psicológico y el asesoramiento de AF, al no tratarse de una actividad dirigida, pudiese favorecer esa reducción tanto del IMC como del porcentaje de masa grasa en la intervención desarrollada en nuestro estudio. Y es que según Aguilar et al., (2014) se confirma el valor de la AF en la reducción del sobrepeso y la obesidad en niños y adolescentes.

Tal y como hemos comentado, sabemos que la AF produce modificaciones en la composición corporal pero también lo hace en la CF (Leppänen et al., 2016), y la mejora de la capacidad cardiorrespiratoria se asocia de manera inversa con los niveles de obesidad en adolescentes (Ortega, Ruiz \& Castillo, 2013).

Nuestros resultados relativos a la capacidad cardiorrespiratoria, medida de manera indirecta a través del último palier completado en el test de CN con un valor de $d$ de Cohen 0.40 para el grupo completo, indican un ligero cambio significativo en el VO2máx de casi un mililitro de oxígeno por kilogramo y minuto. Aunque cada vez parece más evidente la afirmación que el cumplimiento de las recomendaciones de AF actuales puede producir una mejora en la capacidad cardiorrespiratoria, tal y como se refleja en los resultados del estudio de Ortega, Ruiz, Hurting-Wennlöf y Sjöström (2008), en el análisis efectuado de nuestra intervención esa mejora no resulta suficiente para considerar dicho valor de importancia biológica. La relevancia de conseguir una mejora en la CF de los adolescentes gracias al cumplimiento de las recomendaciones de AF, sin necesidad de grande costes ni aparatos, incide tanto en la salud actual como futura. Además, un estilo de vida activo podría reducir las consecuencias negativas de una dieta poco saludable (Cuenca-Garcia et al., 2012), y las consecuencias asociadas al exceso de peso se pueden contrarrestar con altos índices de capacidad cardiorrespiratoria (Castro-Pinero et al., 2012). Por otra parte, el nivel de capacidad cardiorrespiratoria está asociado de manera negativa con los niveles de grasa corporal presentes y futuros (Ortega et al., 2013). Una intervención de AF y de mayor duración y/o intensidad podría incrementar los resultados que, aunque 
tenues, son ya positivos en la capacidad cardiorrespiratoria de los adolescentes participantes en nuestro estudio.

Respecto a la fuerza muscular, evaluada a través del salto horizontal y la dinamometría manual, no se han apreciado mejoras. En el caso de la fuerza prensora de mano y con un valor de tamaño del efecto pequeño, los resultados determinan una pérdida de fuerza, que es solo significativa en chicas. Respecto a la fuerza explosiva del tren inferior no hubo modificación de los valores respecto a la línea base. Ardoy et al., (2011), cuya intervención se realizó a lo largo de 16 semanas en un centro educativo en el que participaron 67 adolescentes entre 12 y 14 ańos, hallaron que incrementar la frecuencia semanal de EF produjo mejoras en la CF, principalmente de la capacidad cardiorrespiratoria pero no así en la fuerza muscular. Nuestro estudio se centró sobre todo en el incremento de la capacidad cardiorrespiratoria y aunque un incremento de fuerza se hubiera entendido debido al desarrollo biológico de la etapa en la que se encuentran, factores como la motivación, pudieron, entre otros, dar como consecuencia los resultados encontrados. Así, Alberga et al., (2016) al examinar los efectos de un entrenamiento dirigido aeróbico, de fuerza, o combinado, de veintidós semanas de duración en 304 adolescentes de 14 a 18 ańos de edad con obesidad, con un percentil igual o por encima del 85 , observó que la mayor mejora sobre la capacidad cardiorrespiratoria se obtuvo tras el entrenamiento de la resistencia, mientras que el trabajo de fuerza y combinado lo tuvo sobre la fuerza muscular y la resistencia.

Aún hoy en día, es difícil determinar el tratamiento más eficaz a la hora de abordar el problema de sobrepeso y obesidad en los adolescentes dada la multitud de variables que pueden afectar a la eficacia del programa, aunque el componente AF ratifique su inclusión en estudio de intervención que tenga dicho objetivo. Nuestro estudio tenía como finalidad observar cómo un estudio multicomponente en el que se incluía asesoramiento sobre la AF podía influir en la cantidad de AF realizada por un grupo de adolescentes con sobrepeso y obesidad. Parece que el asesoramiento sobre AF puede ser eficaz en la prevención del sobrepeso o la obesidad (Bonsergent et al., 2013). Y que la realización de AF, independientemente de la intensidad y de la frecuencia semanal, por personas inactivas genera beneficios sobre su salud (Tremblay et al., 2011). Poitras et al., (2015) muestran una tendencia a que la AF total y no sólo la AFMV, establezca relaciones positivas con marcadores positivos de salud y con modificaciones en la aptitud física, pudiendo disminuir el riesgo de padecer sobrepeso u obesidad (Brown, Kelly \& Summerbell, 2007). Es importante, por tanto, que los programas de intervención favorezcan el incremento de AF (Moreno \& Gracia-Marco, 2012). Así, Alberga et al., (2012) proponen una serie de etapas para el tratamiento del sobrepeso y la obesidad en adolescentes que van desde la prevención hasta la cirugía. Según la revisión de la literatura realizada por este grupo, el ejercicio aeróbico con entrenamiento de fuerza puede ser el más efectivo en términos de pérdida y grasa, y una vez que se pasa a la etapa de intervención, el tratamiento multicomponente que incluya AF debe ser dirigido. Según otro análisis de estudios realizado por De Miguel-Etayo, Bueno, Garagorri y Moreno (2013) lo importante es conseguir un balance energético negativo y a la intervención dietética se debe sumar la intervención de la AF y/o las psicológicas para provocar cambios de comportamiento. La eficacia parece también estar demostrada cuando se combina la AF con la orientación nutricional en la reducción del peso y el IMC en adolescentes según el reconocimiento de estudios realizado por Rocha-Silva, Martín-Matillas, Carbonell-Baeza y Delgado-Fernández (2014).

\section{Limitaciones del estudio y perspectivas de futuro}

No hubo grupo control y no se valoró la maduración sexual de los adolescentes a través del desarrollo físico de los mismos mediante la escala de Tanner o similar, por tanto, es difícil diferenciar qué efecto se debe al propio programa o al crecimiento somático de los participantes. Además, no se puede determinar con exactitud cuánto efecto está ocasionado por asesoramiento en AF del programa y cuánto al resto de componentes implicados, es decir, a las recomendaciones alimentarias y control dietético, y al apoyo psicológico. Por otro lado, la muestra es pequeña y tampoco se realizó una medida de seguimiento tras finalizarse el programa. Y, por último, aunque la duración del programa entra dentro de los márgenes que se contempla en la evidencia científica, podría considerarse escasa para generar cambios a nivel de CF.

\section{Conclusiones}

El presente estudio centra su atención en la AF, encontrándose inmerso en un proyecto multidisciplinar cuyo objetivo era modificar los estilos de vida relacionados con la alimentación y mejorar las habilidades cognitivas conductuales y la AF en un grupo de adolescentes con sobrepeso y obesidad granadinos. El control de la alimentación, el trabajo psicológico y el asesoramiento para cumplir con las recomendaciones de realizar 300 minutos de AF semanal ha generado en los adolescentes con exceso de peso reducción del IMC y del porcentaje de grasa corporal, así como cambios de poca significación biológica, aunque positivos en la condición física cardiorrespiratoria.

\section{Financiación}

Estudio BRAINOBE, financiado por Consejería de Salud de la Junta de Andalucía (PI 0416/2008). 


\section{Referencias}

1. Aguilar, M. J., González, E., García, C. J., García, P. A., Álvarez, J., Padilla, C. A., . . O Ocete, E. (2011). Obesity in a school children population from Granada: assessment of the efficacy of an educational intervention. Nutrición Hospitalaria, 26(3), 636-641. doi:10.1590/S021216112011000300029

2. Aguilar, M. J., Oregón, A., Baena, L., Noack, P. J., Levet, M. C., \& Sánchez, A. M. (2015). Efecto rebote de los programas de intervención para reducir el sobrepeso y la obesidad de niños y adolescentes; revisión sistemática. Nutrición Hospitalaria, 32(6), 2508-2517.

3. Aguilar, M. J., Ortegón, A., Mur, N., Sánchez, J. C., García, J. J., García, I., \& Sánchez, A. M. (2014). Programas de actividad física para reducir sobrepeso y obesidad en niños y adolescentes; revisión sistemática. Nutrición Hospitalaria, 30(4), 727-740.

4. Ainsworth, B. E., Haskell, W. L., Herrmann, S. D., Meckes, N., Basset JR., D. R., Tudor-Locke, C., . . . Leon, A. (2011). 2011 Compendium of PA: a second update of codes and met values. Medice \& Science in Sports \& Exercise, 43(8), 1575-1581.

5. Ainsworth, B. E., Willian, L., Haskell, Whitt, M. C., Irwin, M. L., Swartz, A. M., . . L Leon, S. L. (2000). Compendium of Physical Activities: an update of activity codes and MET intensities. Medicine \& Science in Sports \& Exercise, 32(9), S498-S516.

6. Alberga, A. S., Prud'homme, D., Sigal, R. J., Goldfield, G. S., Hadjiyannakis, S., Phillips, P., . . Kenny, G. P. (2016). Effects of aerobic training, resistance training, or both on cardiorespiratory and musculoskeletal fitness in adolescents with obesity: the HEARTY trial. Appl Physiol Nutr Metab, 41(3), 255-265. doi:10.1139/apnm-2015-0413

7. Alberga, A. S., Sigal, R. J., Goldfield, G., Prud'homme, D., \& Kenny, G. P. (2012). Overweight and obese teenagers: why is adolescence a critical period? Pediatr Obes, 7(4), 261-273. doi:10.1111/j.20476310.2011.00046.x

8. Ardoy, D. N., Fernández, J. M., Ruiz, J. R., Chillón, P., España, V., Castillo, F. M., \& Ortega, F. B. (2011). Improving Physical Fitness in Adolescents Through a School-Based Intervention: the EDUFIT Study. Revista Española de Cardiología, 64(6), 484-491.

9. Bonsergent, E., Agrinier, N., Thilly, N., Tessier, S., Legrand, K., Lecomte, E., . . G Group, P. T. (2013). Overweight and obesity prevention for adolescents: a cluster randomized controlled trial in a school setting. Am J Prev Med, 44(1), 30-39. doi:10.1016/j.amepre.2012.09.055

10. Brown, T., Kelly, S., \& Summerbell, C. (2007). Prevention of obesity: a review of intervention. Obesity, 8(1), 127-130.

11. Brown, T., \& Summerbell, C. (2009). Systematic review of schoolbased interventions that focus on changing dietary intake and physical activity levels to prevent childhood obesity: an update (actualización) to the obesity guidance produced by the National Institute for Health and Clinical Excellence. Obesity, 10, 110-141.

12. Castillo, I., \& Balaguer, I. (2002). Actividad física, ejercicio físico y deporte en la adolescencia temprana. In I. Balaguer (Ed.), Estilos de vida en la adolescencia (pp. 37-64). Valencia.

13. Castro-Pinero, J., Padilla-Moledo, C., Ortega, F. B., Moliner-Urdiales, D., Keating, X., \& Ruiz, J. R. (2012). Cardiorespiratory fitness and fatness are associated with health complaints and health risk behaviors in youth. J Phys Act Health, 9(5), 642-649.

14. Cole, T. J., Bellizi, M. C., Flegal, K. F., \& Dietz, W. H. (2000). Establishing a standard definition for child overweight and obesity worldwide: international survey. Bmj.

15. Cuenca-Garcia, M., Ortega, F. B., Ruiz, J. R., González-Gross, M. Labayen, I., Jago, R., . . group, H. s. (2012). Combined influence of healthy diet and active lifestyle on cardiovascular disease risk factors in adolescents. Scand J Med Sci Sports, 24(3), 553-562. doi:10.1111/ sms. 12022
16. DeBar, L.L., Stevens, V.J., Perrin, N., Wu, P., Pearson, J., Yarborough, B.J., Dickerson, J., Lynch, F., (2012). Pediatrics 129(3), e611-20.

17. de Miguel-Etayo, P., Moreno, L. A., Iglesia, I., Bel-Serrat, S., Mouratidou, T., \& Garagorri, J. M. (2013). Body composition changes during interventions to treat overweight and obesity in children and adolescents; a descriptive review. Nutr Hosp, 28(1), 52-62. doi:10.3305/ nh.2013.28.1.6264

18. Delgado-Rico, E., Rio-Valle, J. S., Gonzalez-Jimenez, E., Campoy, C., \& Verdejo-Garcia, A. (2012). BMI predicts emotion-driven impulsivity and cognitive inflexibility in adolescents with excess weight. Obesity (Silver Spring), 20(8), 1604-1610. doi:10.1038/oby.2012.47

19. Dos Santos, E., Gonçalves, E. M., Morcillo, A. M., Guerra-Júnior, G. \& Amancio, O. M. (2015). Effects of programmed physical activity on body composition in post-pubertal schoolchildren. J Pediatr (Rio J), 91(2), 122-129. doi:10.1016/j.jped.2014.06.004

20. Evans, R. K., Franco, R. L., Stern, M., Wickham, E. P., Bryan, D. L., Herrick, J. E., . . . Laver, J. H. (2009). Evaluation of a 6-month multi-disciplinary healthy weight management program targeting urban, overweight adolescents: effects on physical fitness, physical activity, and blood lipid profiles. Int J Pediatr Obes, 4(3), 130-133. doi:10.1080/17477160802314997

21. Fernández, J. A., \& Hoyos, L. A. (2015). Perspectiva actual de la pro moción de actividad física para la salud en niños y adolescentes en el contexto escolar. Revisión. Lúdica pedagógica, 22(2015-11), 85-99.

22. Gao, Z., Novick, M., Muller, M.D., Williams, R.J., Spilk, S., Leuenberger, U.A., Sinoway, L.I., (2013). Exercise and diet-induced weight loss attenuates oxidative stress related-coronary vasoconstriction in obese adolescents. Eur J Appl Physiol 113(2), 519-28.

23. García, A., Burgueño, R., López, D., \& Ortega, F. B. (2013). Condición física, adiposidad y autoconcepto en adolescentes. Estudio piloto. Revista de Psicología del Deporte, 22, 453-461.

24. Ghatrehsamani, S., Khavarian, N., Beizaei, M., Ramedan, R., Poursafa, P., \& Kelishadi, R. (2010). Effect of different physical activity training methods on overweight adolescents. ARYA Atheroscler, 6(2), 45-49.

25. Graf, C., Beneke, R., Bloch, W., Bucksch, J., Dordel, S., Eiser, S., . Woll, A. (2014). Recommendations for promoting physical activity for children and adolescents in Germany. A consensus statement. Obes Facts, 7(3), 178-190. doi:10.1159/000362485

26. Lee, Y. H., Song, Y. W., Kim, H. S., Lee, S. Y., Jeong, H. S., Suh, S. H., . Hong, Y. M. (2010). The efects of an exercise program on antropometric, metabolic, and cardiovascular parameters in obese children. The Korean Society of Cardiology, 40, 179-184.

27. Leppänen, M. H., Nyström, C. D., Henriksson, P., Pomeroy, J., Ruiz, J. R., Ortega, F. B., . . L Löf, M. (2016). Physical activity intensity, sedentary behavior, body composition and physical fitness in 4-year-old children: results from the minister trial. International Journal of Obesity, 38, S144-S151. doi:10.1038/ijo.2016.54

28. Lopez, L., Audisio, Y., \& Berra, S. (2010). Effectiveness of populationbased interventions on the prevention of overweight in children and adolescents. Med Clin (Barc), 135(10), 462-469. doi:10.1016/j.medcli.2009.06.010

29. Lèger, L. A., Mercier, D., Gadoury, C., \& Lambert, J. (1988). The multistage 20 metre shuttle run test for aerobic fitness. J Sports Sci, 6(2), 93-101. doi:10.1080/02640418808729800

30. López, G. F., López, J. N., \& Diaz, A. (2016). Efectos de un programa de actividad física intensa en la composición corporal de adolescentes murcianos. Revista Euroamericana de Ciencias del Deporte, 5(2), 83-88.

31. Martin-Matillas, M., Ortega, F. B., Ruiz, J. R., Martinez-Gomez, D., Vicente-Rodriguez, G., Marcos, A., . . . Helena Study, G. (2012). Active relatives and health-related physical fitness in European adolescents: 
the HELENA Study. J Sports Sci, 30(13), 1329-1335. doi:10.1080/026 40414.2012.710758

32. Martínez, A., Mayorga, D., \& Viviana, J. (2016). Relación de los niveles de actividad física con el género y el perfil de riesgo cardiovascular en adolescentes granadinos. Implicaciones didácticas para la educación física. Revista de curriculum y formación del profesorado, 20(1).

33. Martínez, D., Welk, G. J., Calle, M. E., Marcos, A., Veiga, O. L., \& Group, t. A. S. (2009). Preliminary evidence of physical activity levels measured by accelerometer in Spanish adolescents; The AFINOS Study. Nutrición Hospitalaria, 24(2), 226-232.

34. Martínez Vizcaíno, V., Salcedo Aguilar, F., Franquelo Gutiérrez, R., Solera Martínez, M., , Sánchez López, M., López García, E., and Rodríguez Artalejo, F., (2008). Assessment of an after-school physical activity program to prevent obesity among 9 - to 10 -year-old children: a cluster randomized trial. International Journal of Obesity, 32, 12-22.

35. Martínez, Vizcaíno, V., \& Sánchez- López, M. (2008). Relación entre actividad física y condición física en niños y adolescentes. Revista Española de Cardiología, 61(02), 108-111.

36. Martínez-Gómez, D., Gómez-Martínez, S., Puertollano, M. A., Nova, E., Wärnberg, J., Veiga, O. L., . . group, E. S. (2009). Design and evaluation of a treatment programme for Spanish adolescents with overweight and obesity. The EVASYON study. BMC Public Health 9 (414).

37. Mayorga-Vega, D., Merino-Marban, R., \& Rodríguez-Fernández, E. (2013). Relación entre la capacidad cardiorrespiratoria y el rendimiento en los test de condición física relacionada con la salud incluidos en la batería ALPHA en nińos de 10-12 años. Cultura, Ciencia y Deporte, 8, 41-47.

38. Menor, M. J., Aguilar, M. J., Mur, N., \& Santana, C. (2017). Efectividad de las intervenciones educativas para la atención de la salud. Revisión sistemática. Medisur, 15(1).

39. Morales, P.F., Sánchez-López, M., Moya-Martínez, P., García-Prieto, J.C., Martínez-Andrés, M., García, N.L., Martínez-Vizcaíno, V., (2013). Health-related quality of life, obesity, and fitness in schoolchildren: the Cuenca study. Qual Life Res, 22 (7), 1515-23.

40. Moreno, L. A., \& Gracia-Marco, L. (2012). [Obesity prevention from physical activity: from theoretical discourse to practice]. An Pediatr (Barc), 77(2), 136.e131-136. doi:10.1016/j.anpedi.2012.04.011

41. Moreno, L. A., Mesana, M. I., Gonzalez-Gross, M., Gil, C. M., Fleta, J., Warnberg, J., . . Bueno, M. (2006). Anthropometric body fat composition reference values in Spanish adolescents. The AVENA Study. Eur J Clin Nutr, 60(2), 191-196. doi:10.1038/sj.ejcn.1602285

42. Nakagawa, S., \& Cuthill, I. C. (2007). Effect size, confidence interval and statistical significance: a practical guide for biologists. Biol Rev Camb Philos Soc, 82(4), 591-605. doi:10.1111/j.1469-185X.2007.00027.x

43. OECD. (2012). Health at a Glance: Europe 2012: OECD Publishing.

44. Ortega, F. B., Artero, E. G., Ruiz, J. R., Espana-Romero, V., JimenezPavon, D., Vicente-Rodriguez, G., . . . study, H. (2011). Physical fitness levels among European adolescents: the HELENA study. Br J Sports Med, 45(1), 20-29. doi:10.1136/bjsm.2009.062679

45. Ortega, F.B., Labayen, I., Ruiz, J.R., Kurvinen, E., Loit, H.M., Harro, J., Veidebaum, T., \& Sjöström, M. (2011). Improvements in fitness reduce the risk of becoming overweight across puberty. Med Sci Sport Exerc, 43(10), 1891-7

46. Ortega, F. B., Ruiz, J. R., \& Castillo, M. J. (2013). Physical activity, physical fitness, and overweight in children and adolescents: evidence from epidemiologic studies. Endocrinología y Nutrición, 60(8), 458-469. doi:10.1016/j.endonu.2012.10.006

47. Ortega, F. B., Ruiz, J. R., Castillo, M. J., Moreno, L. A., GonzálezGross, M., Warnberg, J., ... Group, A. (2005). Low level of physical fitness in spanish adolescents. Relevance for future cardiovascular health (Avena study). Rev Esp Cardiol (Engl Ed), 58(8), 898-909. doi:10.1016/ s1885-5857(06)60372-1
48. Ortega, F. B., Ruiz, J. R., Castillo, M. J., \& Sjöström, M. (2008). Physical fitness in childhood and adolescence: a powerful marker of health. International Journal Obesity (Lond), 32(1), 1-11. doi:10.1038/ sj.ijo.0803774

49. Ortega, F. B., Ruiz, J. R., Hurtig, A., \& Sjöström, M. (2008). Physically active adolescents are more likely to have a healthier cardiovascular fitness level independently of their adiposity status. The European youth heart study. Revista Española de Cardiología, 61(2)(2), 123-129.

50. Ortega, F. B., Sanchez-Lopez, M., Solera-Martinez, M., FernandezSanchez, A., Sjostrom, M., \& Martinez-Vizcaino, V. (2013). Selfreported and measured cardiorespiratory fitness similarly predict cardiovascular disease risk in young adults. Scand J Med Sci Sports, 23(6), 749-757. doi:10.1111/j.1600-0838.2012.01454.x

51. OMS (2014). Informe sobre la situación mundial de las enfermedades no transmisibles. OMS.

52. OMS (2016). Recuperado de http://www.who.int/mediacentre/factsheets/fs311/es/

53. Pastor, J. C., Gil, P., Tortosa, M., \& Martínez, J. (2012). Efectos de un programa de actividad física extracurricular en nińos de primer ciclo de ESO con sobrepeso y obesidad. Revista de Psicología del Deporte, 21, 379-385.

54. Patrick, K., Norman GJ, Calfas KJ, et al. Diet, Physical Activity, and Sedentary Behaviors as Risk Factors for Overweight in Adolescence. Arch Pediatr Adolesc Med. 2004;158(4):385-390

55. Poitras, V. J., Gray, C. E., Borghese, M. M., Carson, V., Chaput, J. P., Cansen, I., . . . Tremblay, M. S. (2016). Systematic review of the relationships between objectively measured physical activity and health indicators in school-aged children and youth. Applied Physiology, Nutrition, and Metabolism, 41, S197-S239.

56. Prochaska, J. J., Sallis, J. F., \& Long, B. (2001). A physical activity screening measure for use with adolescents in primary care. Arch Pediatr Adolesc Med, 155(5), 554-559.

57. Rajmil, L., Bel, J., Clofent, R., Cabezas, C., Castell, C., \& Espallargues, M. (2016). Intervenciones clínicas en sobrepeso y obesidad: revisión sistemática de la literatura 2009-2014. Anales de pediatría.

58. Ramos, P., Jiménez, A., Rivera, F., \& Moreno, C. (2016). Evolución de la práctica de la actividad física en los adolescentes españoles. Revista Internacional de Medicina y Ciencias de la Actividad Física y el Deporte, 16(62).

59. Roberts, C. K., Izadpanah, A., Angadi, S. S., \& Barnard, R. J. (2013). Effects of an intensive short-term diet and exercise intervention: comparison between normal-weight and obese children. Am J Physiol Regul Integr Comp Physiol, 305(5), R552-557. doi:10.1152/ajpregu.00131.2013

60. Rocha Silva, D., Martín-Matillas, M., Carbonell-Baeza, A., Aparicio, V. A., \& Delgado-Fernández, M. (2014). Efectos de los programas de intervención enfocados al tratamiento del sobrepeso/obesidad infantil adolescente. Revista Andaluza de Medicina del Deporte, 7(1), 33-43.

61. Ruiz, J.R., Castro-Pinero, J., Artero, E.G., Ortega, F.B., Sjöström, M., Suni, J., Castillo, M.J. (2009). Predective validity of health-related fitness in youth: a systematic review. Br J Sports Med, 43(12), 909-23.

62. Ruiz, J. R., España, V., Castro, J., Artego, E. G., Ortega, F. B., \& Cuenca, M. (2011). Manual de instrucciones batería ALPHA-Fitness. Nutrición Hospitalaria, 26(6).

63. Ruiz, J. R., Rizzo, N. S., Hurtig, A., Ortega, F. B., Warnberg, J., \& Sjöström, M. (2006). Relations of total physical activity and intensity to fitness and fatness in children: the European Youth Heart Study. The American Journal of Clinical Nutrition, 84(2), 299-303.

64. Siwik, V., Kutob, R., Ritenbaugh, C., Cruz, L., Senf, J., Aickin, M., . . . Shatte, A. (2013). Intervention in overweight children improves body mass index (BMI) and physical activity. J Am Board Fam Med, 26(2), 126-137. doi:10.3122/jabfm.2013.02.120118

65. Summerbell, C. D., Waters, E., Edmunds, L. D., Kelly, S., Brown, T., \& Campbell, K. J. (2005). Interventions for preventing obesity in chil- 
dren. Cochrane Database Syst Rev(3), Cd001871. doi:10.1002/14651858. CD001871.pub2

66. Tremblay, M. S., Carson, V., Chaput, J. P., Connor Gorber, S., Dinh, T., Duggan, M., . . . Zehr, L. (2016). Canadian 24-Hour Movement Guidelines for Children and Youth: An Integration of Physical Activity, Sedentary Behaviour, and Sleep. Appl Physiol Nutr Metab, 41(6 Suppl 3), S311-327. doi:10.1139/apnm-2016-0151

67. Vanhelst, J., Fardy, P. S., Béghin, L., Bui-Xuan, G., \& Mikulovic, J. (2015). Strategies in intervention programmes for obese youth: implication of the age and the type of physical activities. Clin Physiol Funct Imaging, 35(1), 17-20. doi:10.1111/cpf.12112

68. Vasques, C., Magalhaes, P., Cortinhas, A., Mota, P., Leitao, J., \& Lopes, V. P. (2014). Effects of intervention programs on child and adolescent BMI: A meta-analysis study. J Phys Act Health, 11(2), 426-444. doi:10.1123/jpah.2012-0035

69. Verloigne, M., Van Lippevelde, W., Maes, L., Yildirim, M., Chinapaw, M., Manios, Y., . . . De Bourdeaudhuij, I. (2012). Levels of physical activity and sedentary time among 10- t0 12-year-old boys and girls across \% European countries using accelerometers: an observational study within the ENERGY-project. International Journal of Behavioral Nutrition and Physical Activity, 9(34).

70. Waters, E., de Silva-Sanigorski, A., Hall, B. J., Brown, T., Campbell, K. J., Gao, Y., . . S Summerbell, C. D. (2011). Interventions for preventing obesity in children. Cochrane Database Syst Rev(12), CD001871. doi:10.1002/14651858.CD001871.pub3

71. WHO. (2010). Global recommendations on physical activity for health. https://www.who.int/publications/i/item/9789241599979 